\title{
The edge of an event: Invariants of a moving illusory contour
}

\author{
VICTOR KLYMENKO and NAOMI WEISSTEIN \\ State University of New York, Buffalo, New York
}

\begin{abstract}
The motion-induced contour is an illusory contour produced between two vertices of an object undergoing the figural transformations of rotation-in-depth (Klymenko \& Weisstein, 1980). Although it is not physically present, it is seen at the intersection between the two surfaces of an object where a dihedral edge would normally be located. This illusory contour can be seen despite certain temporal and spatial manipulations of the rotating object. It is perceived during apparent rotation in the intermittent object; and it is also seen in an oblique object where the vertices are not parallel. Of the transformations tested, only rotation-in-depth will produce the motion-induced contour (Klymenko \& Weisstein, 1981); however, it is shown here that this is not sufficient. Two complementary factors are necessary. The transformation of rotation-indepth must specify the structural invariant of a rigid dihedral edge.
\end{abstract}

Klymenko and Weisstein (1980) demonstrated an illusory contour-the motion-induced contourwhich is produced by a particular type of motionrotation-in-depth. When the outline projection of a rectangular solid object tilted towards the observer is rotated about the axis of tilt, an edge is perceived (see Figure 1). A moving illusory contour is seen extending across the empty region between the vertices of the object.

In addition, Klymenko and Weisstein (1981) have reported the following. The lines not constituting the vertices, labeled with *s in Figure 1, are not necessary for perception of the motion-induced contour. Here the term "apex" is defined as the point where the two straight lines constituting each vertex meet. This illusory contour can still be seen if the apices of the object are stationary with respect to the retina when the object undergoes rotation-in-depth. Thus, even in the absence of (absolute) motion of the apices, the figural transformations per se can induce this illusory contour.

In the object rotating in depth in which only two disconnected vertices are present (see Figure 1), the vertices are perceived as part of the same figure, as described by the law of common fate (Wertheimer, 1923). The motion-induced contour is seen connecting the two apices of the vertices of this figure. It extends across space and is seen only during figural transformations over time. The goal of the research described here was to investigate the spatiotemporal determinants of the motion-induced contour.

The stimuli used here were the simplest figures that produce the motion-induced contour-a pair

The authors' mailing address is: Department of Psychology, State University of New York, Buffalo, New York 14226.

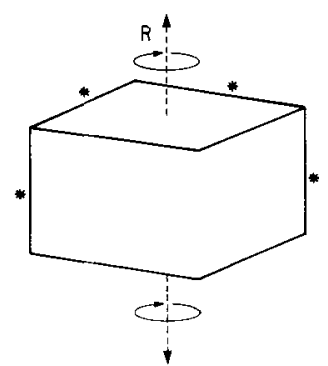

Figure 1. A rectangular solid object rotating about an axis tilted with respect to the picture plane $(R=$ rotation axis). The dihedral edge between the two lower surfaces, although not physically present, is seen during rotation-in-depth. The lines labeled with asterisks are not necessary for the perception of the illusory contour.

of vertices. Several temporal and spatial variations of the vertices were tested in four experiments.

\section{GENERAL METHOD}

The equipment, design and procedure, and data analysis were the same in the four experiments.

\section{Observers}

Seventy naive undergraduates, 67 fulfilling a course requirement and 3 volunteers, took part in the experiments. Twenty-five observers took part in the third experiment and 15 observers took part in each of the other experiments.

\section{Equipment and Displays}

The stimuli used in the experimental conditions were animations presented by a Digital Equipment Corporation PDP-11/ GT -40 computer graphics system. The GT-40 vector graphics display processor draws lines, approximately $1 \mathrm{~mm}$ in width, on a CRT (P-31 phosphor) between coordinates specified by the computer. 
The stimuli consisted of bright lines against a dark background. The luminance of the lines in the stimuli was approximately $19.9 \mathrm{~cd} / \mathrm{m}^{2}$; and the luminance of the fixation markers was approximately $4.5 \mathrm{~cd} / \mathrm{m}^{2}$.

Each graphics display contained five stimuli, the experimental conditions, evenly spaced about a circular array. Each stimulus consisted of a pair of vertices in an animation sequence. Stationary fixation markers were present above and below each stimulus, as shown in Figure 2.

The radius of the circular array, the distance from the center of the display to the position midway between each pair of fixation markers, was $6.47 \mathrm{~cm}$. The distance between each pair of fixation markers was $4.71 \mathrm{~cm}$. The point where the two lines of each vertex meet is referred to as the apex. In the picture plane, the distance between the apices of each figure was $2.1 \mathrm{~cm}$. This distance was the same for all the stimuli and remained constant throughout the animation sequences. For all the experimental conditions, there was never any physical stimulation in the location directly between the fixation markers.

Each display contained five animation sequences which were simultaneously present. In each experimental display, the frames were presented rapidly in succession, $50 \mathrm{msec}$ each, and the set of frames was cycled through repeatedly. Each frame of the display was drawn in approximately $2 \mathrm{msec}$.

The stimuli are classified into three groups as follows. When the animation does not involve three-dimensional motion, the stimulus is referred to as a "pattern." When the animation involves three-dimensional motion of a flat figure, the stimulus is referred to as a "chevron"; and when the animation involves three-dimensional motion of a configuration in space which is not flat, the term "object" is used. The stimuli were displayed under parallel projection. The geometrical description of the stimuli and the temporal transformations across frames are given in the individual method sections of the experiments.

\section{Design and Procedure}

In each experiment, each observer saw one graphics display containing the five stimuli. Each stimulus was located between one of the five pairs of fixation markers, as shown in Figure 2.

For each experiment, the locations of each of the five stimuli in the displays were randomized across observers so that an equal number of observers saw each stimulus in each location. This was done by generating a $5 \times 5$ (location $\times$ stimulus) Latin square for successive sets of five observers.

The observers were tested individually. Each observer was seated in a dark room $1.52 \mathrm{~m}$ from the display screen. A chinrest supported the observer's head such that his eyes were level with the center of the display. The distance between the apices of the stimuli, $2.1 \mathrm{~cm}$, subtended a visual angle of $0.79 \mathrm{deg}$.

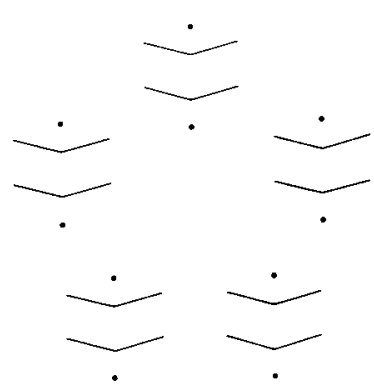

Figure 2. Each experimental display contained five pairs of fixation markers evenly spaced about a circular array. Between each pair of fixation markers there was an animation sequence of a pair of vertices. One of the frames of the display from Experiment 1 is shown. In this frame, in which each pair of vertices is aligned with its respective fixation markers, each stimulus is physically identical in the picture plane.
Before the display was presented, the observer was given instructions defining the task and the use of free magnitude estimation. The observer was told to verbally rate the perceived strength of the "edge" or "line" between the vertices (i.e., the apices) in each of the figures. The observer was instructed to give an arbitrary number to the first figure reported, and to rate each succeeding figure on a proportional basis. Each observer was instructed to fixate directly between the fixation markers above and below each figure, and to make the judgments at an instant when the vertices were aligned with the fixation markers. Alignment refers to the physical condition in which both apices of a figure were directly between the fixation markers. After darkadapting for $5 \frac{1}{2} \mathrm{~min}$, the display was presented and the observer was told to inspect the figures for as long as he or she wished, and to report the ratings in any order by first indicating the location of the figure and then the number assigned to it.

\section{Data Analysis}

Ratings were obtained from each observer for each of the five experimental conditions. The ratings of each observer were weighted equally by normalizing the scores in the following manner. The total sum of each observer's five scores was set equal to 100 by multiplying each individual score by a constant. The mean normalized ratings for the four experiments are given in Tables 1 through 4.

The ratings were analyzed using the repeated measures ANOVA. A posteriori analysis was done using the Newman-Keuls procedure. All experimental conditions that were significant at $p=.05$ were reported.'

Each of the experiments contained a "stationary pattern" to allow comparisons of the various kinds of temporal stimulation with the null condition.

\section{EXPERIMENT 1}

The initial report of the motion-induced contour (Klymenko \& Weisstein, 1981) investigated the temporal conditions necessary for the perception of this illusion. In the first experiment, the basic findings of that report were replicated using a new methodology. Also, a new experimental condition was tested.

In Klymenko and Weisstein's (1981) second experiment there were 10 stimuli that were presented individually in succession. The 10 stimuli included four patterns flickering at different rates, three patterns translating at different speeds, a stationary pattern, and two objects rotating in depth. As compared with the object conditions, the nonobject temporal conditions were ineffective in producing the motion-induced contour. In the present experiment, in which the stimuli were presented simultaneously, four of the five conditions were similar to the conditions in the previous study. These included the three best nonobject conditions and the poorer of the two object conditions. The nonobject conditions included a translating pattern, a flickering pattern, and a stationary pattern. In the previous study, the translating pattern and the object had moved in one direction, whereas in the present experiment these figures moved back and forth between two terminal positions.

Klymenko and Weisstein (1981) found that, of the transformations tested, only rotation-in-depth produced a salient perception of the motion-induced 
contour. The present experiment was designed to determine to what degree rotation-in-depth independently contributes to the perception of the motion-induced contour. The fifth figure in the present experiment, chevron 1 , underwent the same type of motion as the object-rotation-in-depth.

The difference between the object and chevron 1 is the spatial structure given by the rotation sequence. In the chevron, the animation represents a flat surface between the two vertices, whereas in the object two surfaces forming a dihedral edge are specified. Thus, the structural invariant (see Shaw \& Pittenger, 1977), the spatial structure revealed by the transformation of rotation-in-depth, is different in the two conditions.

Observers, using free magnitude estimation, rated five experimental conditions: the stationary pattern, the flickering pattern, the translating pattern, the object, and chevron 1 .

\section{Method}

For each of the five animation sequences, for the frames in which the vertices of each figure were aligned with its fixation markers, each of the figures was physically identical on the screen (see Figure 2). Thus, at the moment when observers made their judgments, the stimuli were identical in the picture plane.

The following sections describe the geometry of the stimuli in the animation sequences. It is useful to visualize the stimuli as being situated in a three-dimensional Cartesian coordinate system as pictured in Figure 3. The $X$ and $Y$ axes represent the horizontal and vertical directions of the picture plane, and the $Z$ axis represents the line of sight in depth of the observer.

Object. One should visualize an outline of a rectangular solid object, the reference object, situated in the Cartesian coordinate system with the point of origin in the center of the object. The four vertical sides of the reference object are parallel to the $Y$ axis; two of the vertical sides intersect the $X$ axis and two intersect the $Z$ axis. The dimensions of the object in three-dimensional space are $2.23 \mathrm{~cm}$ on the $Y$ axis $\times 3.15 \mathrm{~cm} \times 3.15 \mathrm{~cm}$.

One should now visualize an imaginary axis of rotation, the $R$ axis, as being coincident with the $Y$ axis. This $R$ axis, along

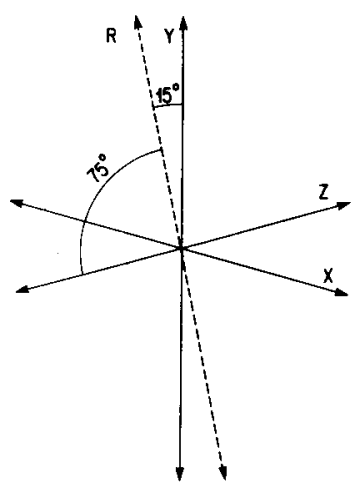

Figure 3. The stimuli can be visualized as situated within a threedimensional Cartesian coordinate system. The $X$ and $Y$ axes represent the horizontal and vertical axes of the picture plane. The $Z$ axis represents the line of sight in depth of the observer. The $\mathbf{R}$ axis, the rotation axis, in the YZ plane is oriented 15 deg with respect to the $Y$ axis. with the reference object, should now be tilted forward 15 deg about the $\mathrm{X}$ axis in the $\mathrm{YZ}$ plane. The $\mathrm{R}$ axis is now in the location shown in Figure 3. The parallel projection of the reference object in the picture plane is shown in Figure 4A. The object condition consists of the four lines of the reference object shown in Figure 4B. This is the 0-deg orientation of the object in the animation sequence shown in Figure $5 \mathrm{~A}$.

In the animation sequence, the object rotated about the $\mathbf{R}$ axis, back and forth, between the far right and far left terminal orientations shown in Figure 5A. The frames in the rotation sequence included the terminal orientations, +40 and $-35 \mathrm{deg}$, and all of the intermediate orientations in steps of $5 \mathrm{deg}$.

Chevron 1. In Figure 4B, one can see, from the spatial description of the object, that the region directly between the vertices represents two surfaces in depth.

Now one should visualize a geometric plane passing through the two apices of the object parallel to the XR plane, as shown in Figure 6. For the chevron, the four lines in Figure 4B lie within the plane, as depicted in Figure 6. Thus, the region directly between the vertices in the chevron represents a single surface in depth.

In the animation sequences, the chevron, in exactly the same manner as the object, rotated about the $R$ axis in steps of 5 deg between, and including, its terminal orientations, +40 and $-35 \mathrm{deg}$, as shown in Figure 5B.

The difference between the chevron and the object is the relative locations of the lines of the figures in three-dimensional space. However, in the 0 -deg orientations of both figures, when the vertices were aligned with the fixation markers, both figures were identical in the picture plane. Also, the apices of both figures move in exactly the same manner throughout the rotation sequence.

Stationary pattern. In this condition, the stimulus shown in Figure 4B was continuously displayed. Each of the frames was the same.

Flickering pattern. In this condition, the stimulus shown in Figure 4B was alternatively present for three frames, then absent for three frames. Thus, the figure flickered at a rate of $31 / 3 \mathbf{~ H z}$.

Translating pattern. In this condition, the stimulus shown in Figure 4B moved horizontally back and forth between two terminal locations, 28 screen units to the left and 32 screen units to the right ( 44.7944 screen units $/ \mathrm{cm})$. The motion sequence included the terminal locations and all of the intermediate locations in steps of 4 screen units.

\section{Results and Discussion}

The mean ratings are shown in Table 1 . The analysis of variance indicated that the experimental condition was highly significant $[F(4,56)=11.08, p<$ $.001]$. A posteriori analysis of the experimental conditions based on the Newman-Keuls procedure $(\mathrm{p}=$ .01) showed that the object was significantly better than any of the other conditions. None of the other pairwise comparisons were significant.

As expected, neither flicker nor translatory motion is any more effective than a stationary pattern in producing the motion-induced contour. The chevron, although it underwent the same type of motion as the object, is equally ineffective. Thus, rotationin-depth per se is not sufficient to produce the motioninduced contour.

In the animation sequences, the two vertices in each figure tend to be seen as part of the same figure as described by common fate, the detection of figure against the ground of space-time. It has been noted how two moving dots, in the absence of information to the contrary, tend to be seen as endpoints of a 


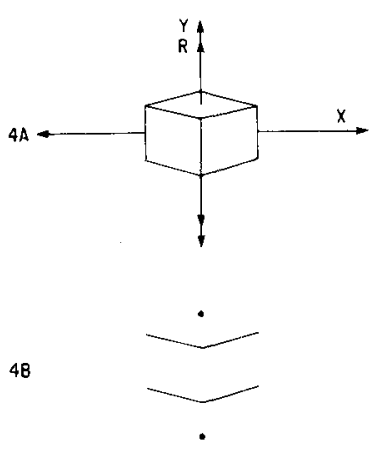

Figure 4. The parallel projection of the 0-deg orientation of the reference object is shown in $4 A$. Only the four lines shown in $4 \mathrm{~B}$ were displayed in the animation sequence of the object.

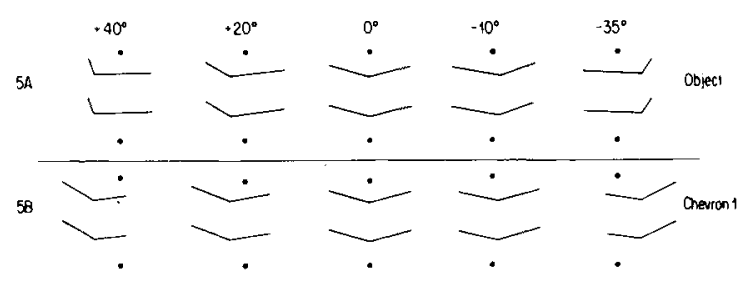

Figure 5. A subset of the frames in the rotation sequence of the object (5A) and chevron 1 (5B). The figures rotated back and forth repeatedly between the two terminal orientations in steps of $5 \mathrm{deg}$. In the 0-deg orientation, the figures were aligned with their respective fixation markers and were identical in the picture plane.

rigid rod (Johansson, 1974); this is the simplest and/ or most stable perceptual organization, or Pragnantz (see Restle, 1979, for a modern analysis). Analogously, when two lines move together, they tend to be seen as the edges of a surface. In the figures tested, the area between each pair of parallel lines may be considered as a surface with an ambiguous orientation in space. In the stationary, translating, and flickering patterns, there is no information that these two surfaces lie in different depth planes. In the chevron, however, there is information that the entire figure lies in the same depth plane. In contrast, in the rotation sequence of the object, there is information that the right and left parts of the figure lie in different depth planes. The junction of these two surfaces defines a rigid dihedral angle. This is the location of the motion-induced contour.

The motion-induced contour is not a subjective contour or a visual phantom. Unlike subjective contours (Schumann, 1904), this illusory contour is not seen in stationary patterns. Unlike visual phantoms, a contour completion effect that can be produced when the appropriate stimulus is translated (Tynan \& Sekuler, 1975) or flickered (Genter \& Weisstein,
1981) or steadily presented to dark-adapted observers (Gyoba, 1983), only rotation-in-depth will produce a salient motion-induced contour. However, rotationin-depth is not sufficient. In the chevron, the rotationin-depth sequence does not specify a dihedral edge between the two apices of the figure. Thus, two complementary factors appear to be necessary: the tranformational invariant of rotation-in-depth and the structural invariant of a rigid dihedral edge.

The next experiment further tested the hypothesis that the rotation-in-depth sequence must specify the structural invariant of a rigid dihedral edge.

\section{EXPERIMENT 2}

Experiment 1 found chevron 1 to be ineffective in producing the motion-induced contour, as compared with the object. The present experiment tested two additional chevrons to control for the possibility that an extraneous factor was responsible for the ineffectiveness of chevron 1.

The figural transformations of chevron 2 are the same as those of chevron 1, except that the translational component of the figure in the picture plane was nullified in order to keep the apices in the same location. The animation sequence of chevron 2 represents a flat figure rotating in depth around an axis that passes through the two apices of the figure, whereas the sequence of chevron 1 represents a flat figure rotating in depth about an axis which does not pass through the figure. Chevron 2 is designed as a control for the possibility that the translatory motion of the apices in the picture plane, and not the structural invariant specified by the rotation sequence, may be responsible for the ineffectiveness of chevron 1 in producing the motion-induced contour.

The rotation sequence of chevron 3 represents a flat figure rotating around a vertical axis which passes through the two apices of the figure. Chevron 1 , chevron 2 , and the object each rotate about an axis that is oriented $15 \mathrm{deg}$ with respect to the

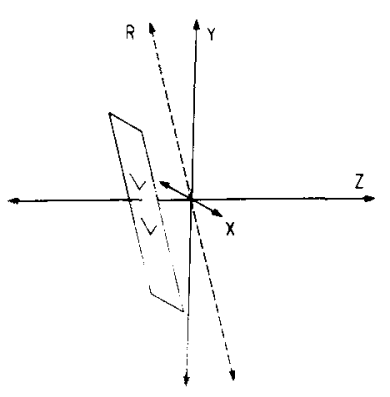

Figure 6. A perspective view of the 0-deg orientation of chevron 1 . The vertices of chevron 1 lie within geometric plane parallel to the XR plane. 
Table 1

Mean Ratings for Experiment 1

\begin{tabular}{ll} 
Object & 33.24 \\
Chevron 1 & 17.17 \\
Translating Pattern & 18.39 \\
Flickering Pattern & 15.61 \\
Stationary Pattern & 15.60 \\
\hline
\end{tabular}

picture plane. On the other hand, chevron 3 rotates around one of the axes of the picture plane, a vertical axis. This figure was included in order to test the effect the orientation of the rotation axis has on the chevrons.

Chevron 2 and chevron 3 test the importance of factors that may be responsible for the ineffectiveness of chevron 1 , other than the lack of specification of a rigid dihedral edge. In the previous experiment, it was hypothesized that the rotation-in-depth sequence must specify the structural invariant of a rigid dihedral edge. In each of the chevrons, the simplest visual interpretation of the motion sequence is that of a rigid flat figure rotation in depth. ${ }^{2}$

The five following experimental conditions were included in the experiment: the stationary pattern, the object, chevron 1 , chevron 2 , and chevron 3 .

\section{Method}

Three of the experimental conditions-the object, chevron 1, and the stationary pattern-were described in the last experiment. In all of the rotation sequences in the present experiment, the terminal orientations were +40 and -40 deg. The following describes the new stimuli.

Chevron 2. The rotation sequence of chevron 2 differed from that of chevron 1 in the following way. During the rotation sequence of chevron 1, the apices moved across the screen so that only when the 0 -deg orientation came into view were the apices aligned with the fixation markers. In chevron 2 , the apices were stationary and aligned with the fixation markers for every orientation. In the rotation sequence, a translational component was nullified to keep the apices in the same location. The relative figural transformations were the same for both conditions. Thus, for chevron 2 , the frames of the rotation sequence were the same as those in Figure $5 B$, with the exception that the vertices were aligned with the fixation markers for every orientation.

This animation sequence can be geometrically represented as follows. The 0-deg orientation of this chevron lies within the XR plane rather than in the parallel plane depicted for chevron 1 in Figure 6. Thus, the axis of rotation, the $R$ axis, passes through the apices of chevron 2.

In the rotation sequence, this chevron rotated around the $\mathbf{R}$ axis in steps of $5 \mathrm{deg}$, between and including the terminal orientations, +40 and -40 deg.

Chevron 3. One should now visualize the stimulus shown in Figure 4B as lying within the $X Y$ plane, with the $Y$ axis passing through the two apices. This represents the 0 -deg orientation of chevron 3, and is the same, in the picture plane, as the 0-deg orientations of the other chevrons and the object.

In the rotation sequence, this chevron rotated around the $Y$ axis in steps of 5 deg between and including the terminal orientations of +40 and -40 deg.

\section{Results and Discussion}

The mean ratings are shown in Table 2. The experimental condition was highly significant $[F(4,56)$
$=12.63, \mathrm{p}<.001]$. A posteriori analysis of the experimental conditions based on the Newman-Keuls procedure $(p=.01)$ showed that the object was better than each of the other conditions. None of the other pairwise comparisons were significant.

As expected, chevron 1 is again not any more effective than the stationary pattern in producing the motion-induced contour. Chevron 2 is equally ineffective. Chevron 2 differs from chevron 1 in that the apices are stationary due to the absence of a translatory component. Therefore, motion of the apices per se is not a factor, since the global figural transformations of both these figures are the same. Chevron 3, which differs from the other two chevrons in that it rotates on an axis in the picture plane, also does not produce a salient motion-induced contour. Each of the chevrons represent a flat figure rotating in depth. Of the four figures rotating in depth, only in the object does the rotation sequence specify a rigid dihedral edge, and only the object is significantly better than the stationary pattern in producing the motion-induced contour between the vertices. Thus, it appears that the transformation of rotationin-depth must specify the structural invariant of a rigid dihedral edge.

The next experiment investigated the effects of structural and temporal manipulations of the object.

\section{EXPERIMENT 3}

The unitary nature of visual space-time is characteristic of many of the phenomena classified under the rubric of event perception (Caelli, Hoffman, \& Lindman, 1978; Johansson, 1978; Johansson, von Hofsten, \& Jansson, 1980; see also Delong, 1981). For example, it has been shown that the perception of velocity is direct and not based upon independent discriminations of spatial and temporal extent (Lappin, Bell, Harm, \& Kottas, 1975). The perception of the motion-induced contour is similar, in this regard, to the perception of velocity, since both rely upon information which exists only within an integral space-time framework. In the discrimination of velocity, the perceptual relation between space and time has been shown for both continuous and apparent motion (Lappin et al., 1975). The present experiment tested the perceptual saliency of apparent motion in producing the motion-induced contour by including an object in apparent motion (ap-

Table 2

Mean Ratings for Experiment 2

\begin{tabular}{ll} 
Object & 34.23 \\
Chevron 1 & 17.35 \\
Chevron 2 & 14.09 \\
Chevron 3 & 18.30 \\
Stationary Pattern & 16.03 \\
\hline
\end{tabular}


parent rotation), the "intermittent object," as one of the stimuli. The intermittent object tests the ability of the visual system to extract the structural invariant specifying a dihedral edge when the figural transformations are perceptually discontinuous.

One way of characterizing a geometry is by distinguishing the properties that are invariant from those that are variant under a group of transformations (Klein, 1939). Since the object sequences are seen under parallel projection, the information specifying each surface of the object should be invariant within the domain of affine transformations (Gans, 1969). The parallelism of lines is one salient property which is invariant under affine transformations. In the object, there are two sets of parallel lines, which remain so throughout the rotation sequence, as one can see in Figure 5A. The importance of this invariant, parallelism of lines, in producing the motioninduced contour is tested in the stimulus referred to as the "oblique object." This stimulus differs from the object in that the two lines defining each surface of the dihedral edge are never, rather than always, parallel throughout the rotation sequence. The oblique object tests the ability of the visual system to pick up the structural invariant of a dihedral edge when the geometric property of parallelism is unavailable.

We also included, among the experimental conditions, the stimulus called the "rotating pattern." This pattern is unlike both the objects and the chevrons, in that the rotation sequence does not produce figural transformations and, consequently, the kinetic depth information for the spatial structure of the figure is absent. Thus, this figure tests the possibility that rotary motion per se produces the contour when kinetic depth information specifying a single surface is absent, rather than when kinetic depth information specifying two surfaces is present.

The five following experimental conditions were tested: the stationary pattern, the rotating pattern, the object, the intermittent object, and the oblique object.

\section{Method}

Two of the experimental conditions, the object and the stationary pattern, have already been described; they were included as boundary conditions. In the rotation sequences in this experiment, the terminal orientations were +40 and $-40 \mathrm{deg}$. The new conditions are described below.

Intermittent object. This condition was the same as the object except for the fact that every second frame in the rotation sequence was blank. In the object, every orientation is presented for $50 \mathrm{msec}$ in succession. In the intermittent object, the figure is absent for every alternate orientation in the motion sequence.

The terminal orientations, +40 and $-\mathbf{4 0} \mathrm{deg}$, as well as every intermediate orientation in steps of $10 \mathrm{deg}$ were present in the rotation sequence. The figure was present for the 0 -deg orientation, in which the vertices were aligned with the fixation markers. The apparent motion parameters were: stimulus duration, $50 \mathrm{msec}$, and interstimulus interval, $50 \mathrm{msec}$.
Oblique object. In describing the object, we have already referred to the reference object located in a three-dimensional Cartesian coordinate space. The reference object is depicted again in solid lines in Figure 7A. One should now visualize the dotted lines in the figure as lying on the two visible surfaces of the reference object. The dotted lines extend from the point where three solid lines meet to the outer edges of the reference object. In threedimensional space, each of the dotted lines meets the outer edges of the reference object $1.07 \mathrm{~cm}$ below the solid line.

These two lines and two of the lines of the reference object are shown in Figure 7B. This is the 0-deg orientation of the oblique object in the rotation sequence shown in Figure 8A.

The oblique object differed from the object in that the lines in the upper vertex were not parallel to the corresponding lines in the lower vertex for any of the frames in the rotation sequence.

The oblique object rotated about the $R$ axis in exactly the same manner as the object. The frames included the terminal orientations, +40 and $-40 \mathrm{deg}$, and all of the intermediate orientations in steps of $5 \mathrm{deg}$.

Rotating pattern. In this condition, the stimulus shown in Figure $4 \mathrm{~B}$ represents the 0 -deg orientation of the rotation sequence shown in Figure 8B. The figure rotated in the picture plane about an imaginary point $1.79 \mathrm{~cm}$ below the upper fixation marker. The rotation sequence included the terminal orientations, +40 and $-40 \mathrm{deg}$, and all of the intermediate orientations in steps of $5 \mathrm{deg}$.

Unlike the other rotation sequences, there were no figural transformations in the picture plane.

\section{Results and Discussion}

The mean ratings are shown in Table 3 . The experimental conditions were significant $[F(4,96)=8.39$, $\mathrm{p}<.001]$. A posteriori analysis of the experimental

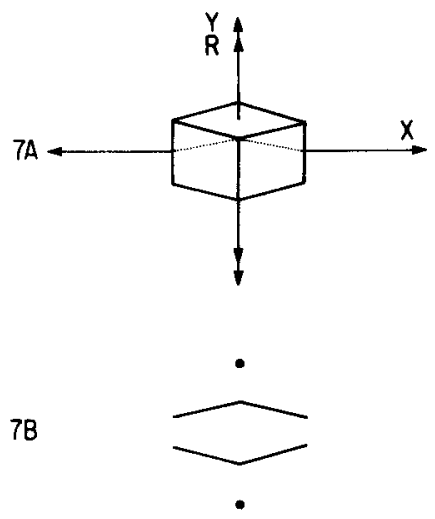

Figure 7. The upper vertex of the oblique object is shown in dotted lines superimposed on the reference object in 7A. The lines of the oblique object are shown in $7 \mathrm{~B}$.

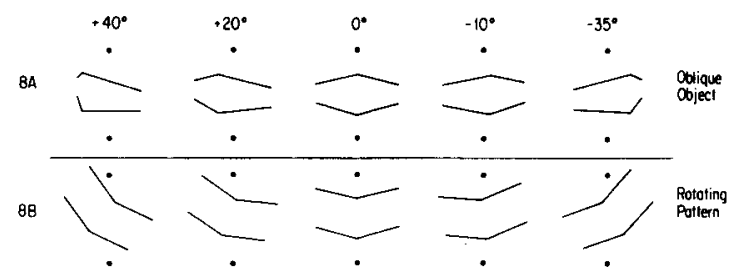

Figure 8. A subset of the frames in the rotation sequence of the oblique object (8A), and the rotating pattern (8B). 
conditions based on the Newman-Keuls procedure showed that both the object and the intermittent object were rated significantly better $(p=.01)$ than each of the two patterns; also, the oblique object was significantly better $(p=.05)$ than the two patterns. None of the other pairwise comparisons were significant.

The rotating pattern is not significantly different from the stationary pattern. Thus, rotation per se, even in the absence of kinetic depth information specifying a flat figure, is not sufficient. This, together with the results of the first two experiments, strengthens the evidence that the rotation sequence must specify a dihedral edge.

The intermittent object is significantly better than the two patterns and not significantly different from the other two objects. Thus, the visual system is able to pick up the structural invariant specifying a dihedral edge under the condition of apparent motion in which the figural transformations are perceptually discontinuous. The lower value of the mean rating of the intermittent object, as compared with that of the object, seems to indicate that the more closely real motion is approximated, the more salient is the perception of the motion-induced contour.

The oblique object, while not significantly different from the object, had a lower mean rating. It is known that subjective contours are diminished or eliminated when real contours are placed so as to intercept the subjective contour. Due to their orientations, the real contours sweep over more of the loci near the apices that are occupied by the illusory contour in the oblique object than they do in the object (compare Figures 5A and 8A). Thus, the real contours, intercepting the locations of the illusory contour over time, may be attenuating its perceptibility relative to the object.

The following experiment further examined the importance of parallelism by comparing the oblique object with oblique patterns and an oblique chevron.

\section{EXPERIMENT 4}

This experiment controlled for parallelism in all of the experimental conditions. The oblique object was compared against figures that were themselves oblique to determine if the differences among the various types of temporal stimulation stand up in the absence of parallelism.

Table 3

Mean Ratings for Experiment 3

\begin{tabular}{ll}
\multicolumn{2}{c}{ Mean Ratings for Experiment 3 } \\
\hline Object & 27.01 \\
Intermittent Object & 24.48 \\
Oblique Object & 21.15 \\
Rotating Pattern & 13.27 \\
Stationary Pattern & 14.11 \\
\hline
\end{tabular}

The same temporal conditions used in the first experiment were represented here by analogous figures: the oblique stationary pattern, the oblique flickering pattern, and the oblique translating pattern. The chevron with the highest mean rating in the second experiment was represented here by a corresponding figure-oblique chevron 3 . This experiment, like the first experiment, again compared an object with four other classes of temporal stimulation, except that this time, all of the figures were oblique.

Thus, this experiment sought to determine if the conclusions of the previous experiments concerning structural and transformational invariants could be generalized to these figures, which do not contain parallel lines.

The five experimental conditions tested were: the oblique stationary pattern, the oblique flickering pattern, the oblique translating pattern, the oblique object, and oblique chevron 3.

\section{Method}

The oblique figure shown in Figure 7B was used in each of the experimental conditions. The oblique object was the same as that described in the last experiment, except that the terminal orientations were +40 and $-35 \mathrm{deg}$; this was also the case for the oblique chevron described below.

Oblique chevron 3. The rotation sequence of this chevron was the same as that described for chevron 3 in the second experiment, except that the stimulus shown in Figure 7B was substituted for the stimulus in Figure 4B.

Figure 7B represents the 0-deg orientation of oblique chevron 3 when the entire figure lies in the picture plane. Oblique chevron 3 rotated around the $\mathbf{Y}$ axis, which passes through its apices, in steps of $5 \mathrm{deg}$ between and including the two terminal orientations, +40 and -35 deg.

Oblique patterns. There were three oblique patterns: the oblique stationary pattern, the oblique flickering pattern, and the oblique translating pattern.

The animation sequences of the oblique patterns were exactly the same as those described for the analogous patterns in the first experiment, except that the stimulus shown in Figure 7B was substituted for the stimulus in Figure 4B.

\section{Results and Discussion}

The mean ratings are shown in Table 4. The experimental condition was significant $[F(4,56)=7.44$, $\mathrm{p}<.001$ ]. A posteriori analysis of the experimental conditions based on the Newman-Keuls procedure $(p=.01)$ showed that the oblique object was significantly better than any of the other experimental conditions. None of the other pairwise comparisons were significant.

Oblique chevron 3, although not significantly different from the oblique patterns, had a higher mean rating. This may be due to the fact that since the apices of this figure were always on the rotation axis, the $Y$ axis, and the lines never crossed over the axis, observers had a strong impression of the rotation 
Table 4

Mean Ratings for Experiment 4

Oblique Object

31.11

Oblique Chevron 3

21.04

Oblique Translating Pattern

15.24

16.93

Oblique Flickering Pattern

Oblique Stationary Pattern

15.67

axis itself. Thus, they tended to rate this figure higher than the patterns.

The oblique object was superior to each of the other stimuli. The geometric property of parallelism is not a necessary factor in specifying the structural invariant of a dihedral edge. Thus, the poorer showing of the oblique object, as compared with the object in Experiment 3, may be due to another factor, such as the interception, over time, of the illusory contour by the real contours. Since parallel lines are not necessary, it seems likely that the motioninduced contour would also appear in rotating objects under polar projection.

The main conclusion of the three previous experiments is that two complementary factors are necessary for the perception of the motion-induced contour: the transformational invariant of rotation-indepth and the structural invariant of a rigid dihedral edge. Parallel lines are not necessary.

\section{GENERAL DISCUSSION}

Of the transformations tested, only rotation-indepth will produce the salient perception of the motioninduced contour (Klymenko \& Weisstein, 1981). Experiments 1 and 2 demonstrated that rotation-indepth is not sufficient to produce this illusory contour. The rotation sequence must specify the structural invariant of a rigid dihedral edge. The motioninduced contour appears when there is information that the region between the vertices consists of two surfaces which lie in different depth planes. It is not seen in the chevrons in which the information specifies one surface between the vertices, nor is it seen in the rotating pattern used in Experiment 3, which had no information about the orientation of the surface or surfaces between the vertices.

Experiment 3 also showed that the motion-induced contour is still seen for certain temporal and spatial manipulations of the object. The illusory edge is seen in the intermittent object, in which the motion sequence is perceptually discontinuous. It is also seen in the oblique object, in which the vertices demarcating the two surfaces are not parallel.

Experiment 4 reaffirmed the conclusions of the first two experiments for the oblique figures: the transformation of rotation-in-depth must specify a rigid dihedral edge.
Klein (1939), in his Erlangen program, categorized various geometries in terms of the structural properties that remain invariant under transformation (see Shaw \& Pittenger, 1977, for a discussion of the application of this to perceptual theory; also see Westheimer, 1978). Recently, particular geometries have been formally applied to describe some of the geometric illusions and figural aftereffects (Watson, 1978), and some phenomena of motion perception (Caelli et al., 1978). In the motion-induced contour, time and space are integral dimensions, since this illusory contour is seen only during figural transformations over time. It is a higher order invariant (Gibson, 1966) of visual space-time. The motioninduced contour is the structural invariant of a rigid dihedral edge revealed by the transformation of rotation-in-depth.

Two complementary factors appear to be necessary: the transformational invariant of rotation-indepth must specify the structural invariant of a rigid dihedral edge. ${ }^{3}$ It is possible that other combinations of transformational and structural invariants may produce salient contours, for example, a translation in depth of the object under polar projection. ${ }^{4}$ This is a question for further research.

The motion-induced contour has been described here as an illusion. However, there is abstract information in the optic array given over time (Gibson, 1979) which specifies the event of a moving dihedral edge.

\section{REFERENCES}

Braunstein, M. L., \& Anderson, G. J. Velocity gradients and relative depth perception. Perception \& Psychophysics, 1981, 29, 145-155.

Caelli, T., Hoffman, W., \& Lindman, T. Subjective Lorentz transformations and the perception of motion. Journal of the Optical Society of America, 1978, 68, 402-411.

Delong, A. J. Phenomenological space-time: Towards an experiental relativity. Science, 1981, 213, 681-683.

Farber, J. M., \& McConkie, A. B. Optimal motions as information for unsigned depth. Journal of Experimental Psychology: Human Perception and Performance, 1979, 5, 494-500.

Gans, D. Transformations and geometries. New York: AppletonCentury-Crofts, 1969.

Genter, C. R., II, \& Weisstein, N. Flickering phantoms: A motion illusion without motion. Vision Research, 1981, 21, 963-966.

GiBson, J. J. The senses considered as perceptual systems. Boston: Houghton Mifflin, 1966.

Gisson, J. J. The ecological approach to visual perception. Boston: Houghton Mifflin, 1979.

GYOBA, J. Stationary phantoms: A completion effect without motion and flicker. Vision Research, 1983, 23, $205-211$.

Harrington, T. L., Harrington, N. K., Wilkins, C. A., \& KoH, Y. O. Visual orientation by motion-produced blur patterns: Detection of divergence. Perception \& Psychophysics, 1981, 28, 298-305.

Johansson, G. Projective transformations as determining visual space perception. In R. B. Macleod \& H. L. Pick, Jr. (Eds.), 
Perception: Essays in honor of James J. Gibson. Ithaca, N.Y: Cornell University Press, 1974.

Johansson, G. Visual event perception. In R. Held, H. W. Leibowitz, \& H. L. Teuber (Eds.), Handbook of sensory physiology (Vol. 8). New York: Springer, 1978.

Johansson, G., von Hofsten, C., \& Jansson, G. Event perception. Annual Review of Psychology, 1980, 31, 27-63.

KAPLAN, G. A. Kinetic disruption of optical texture: The perception of depth at an edge. Perception \& Psychophysics, 1969, 4, 193-198.

KLEIN, F. Elementary mathematics from an advanced standpoint: Geometry. New York: Macmillan, 1939.

KuYMenko, V., \& Weisstein, N. Illusory contour produced by rotation-in-depth. Optics News, 1980, 6, 3, 61. (Abstract)

KLYMenko, V., \& Weisstein, N. The motion-induced contour. Perception, 1981, 10, 627-636.

Lappin, J. S., Bell, H. H., Harm, O. J., \& Kottas, B. On the relation between time and space in the visual discrimination of velocity. Journal of Experimental Psychology: Human Perception and Performance, 1975, 1, 383-394.

REsTLE, F. Coding theory of the perception of motion configurations. Psychological Review, 1979, 86, 1-24.

Rogers, B., \& Graham, M. Motion parallax as an independent cue for depth perception. Perception, 1979, 8, 125-134.

Schumann, F. Einige Beobachtungen über die Zusammenfassung von Gesichtseindrucken zu Einheiten. Psychologische Studien, 1904, 1, 1-32.

Shaw, R., \& Pittengen, J. Perceiving the face of change in changing faces: Implications for a theory of object perception. In R. Shaw \& J. Bransford (Eds.), Perceiving, acting and knowing: Toward an ecological psychology. Hillsdale, N.J: Earlbaum, 1977.

ToDD, J. T. Visual information about rigid and nonrigid motion: A geometric analysis. Journal of Experimental Psychology: Human Perception and Performance, 1982, 8, 238-252.

Tynan, P., \& Se rulen, R. Moving visual phantoms: A new contour completion effect. Science, 1975, 188, 951-952.

Watson, A. A Riemann geometric explanation of the visual illusions and figural after-effects. In E.L.J. Leeuwenberg \& H.J.M.
Buffart (Eds.), Formal theories of visual perception. New York: Wiley, 1978.

WERTHEIMER, M. Untersuchungen zur Lehre von der Gestalt: 2. Psychologische Forschung, 1923, 4, 301-350.

Westheimer, G. Spatial vision and geometrical principles. Journal of the Optical Society of America, 1978, 68, 1370. (Abstract)

\section{NOTES}

1. The following analysis, suggested by Irving Biederman, was also performed. The ratings of each observer were converted to $z$ scores. The $z$ scores were analyzed by the repeated-measures ANOVA, and a posteriori analysis was done using the NewmanKeuls procedure. The results of this analysis were essentially the same in all respects as the analysis based on the normalized ratings.

2. The right and left parts of chevron 2 , chevron 3 , and oblique chevron 3 are sometimes seen as not being rigidly connected and as rotating independently of each other, although synchronously with each other. In this alternative mode, they would form a nonrigid dihedral edge of varying angularity. However, unlike the object, there is no geometric information which specifies a rigid dihedral edge.

3. See Todd (1982) for one formulation of the invariants specifying rigidity in motion perception.

4. Researchers have found that relative depth and contours can be produced by kinetic occlusion (Kaplan, 1969) and by polar projection of horizontal translations (Braunstein \& Anderson, 1981; Farber \& McConkie, 1979; Rogers \& Graham, 1979; also see Harrington, Harrington, Wilkins, \& Koh, 1981). The motioninduced contour differs from these phenomena in that there is no stimulation in the location of the contour. Also, it should be noted that when two frames of the motion sequence of the object are viewed in a stereoscope an equally salient contour will not be induced.

(Manuscript received October 17, 1982; revision accepted for publication March 25, 1983.) 